\title{
Le personnel enseignant et l'enseignement dans l'agenda du Réseau ouest et centre africain de recherche en éducation (ROCARE) : état des lieux vingt ans après Maclure (1997) Teachers and teaching on the agenda of the Educational Research Network for West and Central Africa (ERNWACA): An update twenty years after Maclure (1997) El magisterio y la enseñanza en la agenda de la Red occidental y central africana de investigación en educación (ROCARE): estado actual veinte años después de Maclure (1997)
}

\author{
François Joseph Azoh, Affoué Philomène Koffi et Martial Dembélé
}

\section{Volume 45, numéro 3, hiver 2018}

La quête de l'éducation de qualité pour tous et la profession enseignante dans les pays francophones d'Afrique subsaharienne

URI : https://id.erudit.org/iderudit/1046417ar

DOI : https://doi.org/10.7202/1046417ar

Aller au sommaire du numéro

Éditeur(s)

Association canadienne d'éducation de langue française

ISSN

1916-8659 (numérique)

Découvrir la revue

Citer cet article

Azoh, F. J., Koffi, A. P. \& Dembélé, M. (2018). Le personnel enseignant et l'enseignement dans l'agenda du Réseau ouest et centre africain de recherche en éducation (ROCARE) : état des lieux vingt ans après Maclure (1997). Éducation et francophonie, 45(3), 61-82. https://doi.org/10.7202/1046417ar

\section{Résumé de l'article}

Cet article s'inscrit dans le prolongement de la synthèse réalisée par Maclure en 1997 sur la recherche en éducation en Afrique centrale et occidentale. Il s'agit donc d'une revue des travaux de recherche portant sur le thème du personnel enseignant et de l'enseignement dans les douze pays francophones membres du Réseau ouest et centre africain de recherche en éducation (ROCARE) au cours des vingt dernières années, soit de 1997 à 2017. Cette revue repose sur un corpus documentaire sélectionné dans la bibliothèque numérique du ROCARE. Les 315 études recensées couvrent les quatre sous-thèmes suivants : 1 ) formation du personnel enseignant et pratiques pédagogiques; 2) évaluation des apprentissages et qualité de l'éducation; 3) réformes curriculaires et méthodes pédagogiques; 4) éducation non formelle. Ces études convergent sur le déficit de formation initiale et continue des enseignantes et enseignants, ce qui ne leur permet pas d'appliquer les méthodes pédagogiques et les curriculums issus des réformes et d'offrir un enseignement de qualité. Vingt ans après Maclure, la question enseignante et l'enseignement se présentent dans les mêmes termes.
Tous droits réservés (C) Association canadienne d'éducation de langue française, 2018
Cecument est protégé par la loi sur le droit d'auteur. L'utilisation des services d'Érudit (y compris la reproduction) est assujettie à sa politique d'utilisation que vous pouvez consulter en ligne. 


\section{Le personnel enseignant et l'enseignement dans l'agenda du Réseau ouest et centre africain de recherche en éducation (ROCARE): état des lieux vingt ans après Maclure (1997)}

François Joseph AZOH

École normale supérieure d'Abidjan, Abidjan, Côte d'Ivoire

\section{Affoué Philomène KOFFI}

Université Félix Houphouët-Boigny, Abidjan, Côte d'Ivoire

Martial DEMBÉLÉ

Université de Montréal, Québec, Canada

\section{RÉSUMÉ}

Cet article s'inscrit dans le prolongement de la synthèse réalisée par Maclure en 1997 sur la recherche en éducation en Afrique centrale et occidentale. Il s'agit donc d'une revue des travaux de recherche portant sur le thème du personnel enseignant et de l'enseignement dans les douze pays francophones membres du Réseau ouest et centre africain de recherche en éducation (ROCARE) au cours des vingt dernières 
années, soit de 1997 à 2017. Cette revue repose sur un corpus documentaire sélectionné dans la bibliothèque numérique du ROCARE. Les 315 études recensées couvrent les quatre sous-thèmes suivants: 1) formation du personnel enseignant et pratiques pédagogiques; 2) évaluation des apprentissages et qualité de l'éducation; 3) réformes curriculaires et méthodes pédagogiques; 4) éducation non formelle. Ces études convergent sur le déficit de formation initiale et continue des enseignantes et enseignants, ce qui ne leur permet pas d'appliquer les méthodes pédagogiques et les curriculums issus des réformes et d'offrir un enseignement de qualité. Vingt ans après Maclure, la question enseignante et l'enseignement se présentent dans les mêmes termes.

\section{ABSTRACT}

\section{Teachers and teaching on the agenda of the Educational Research Network for West and Central Africa (ERNWACA): An update twenty years after Maclure (1997)}

François Joseph AZOH, Higher Normal School of Abidjan, Abidjan, Ivory Coast

Affoué Philomène KOFFI, Félix Houphouët-Boigny University, Abidjan, Ivory Coast

Martial DEMBÉLÉ, University of Montreal, Quebec, Canada

This article is an extension of Maclure's 1997 synthesis of educational research in West and Central Africa. It is a literature review on the theme of teachers and teaching in the twelve Francophone member countries of the Educational Research Network for West and Central Africa (ERNWACA) over the past twenty years, from 1997 to 2017. This review is based on a set of documents selected in ERNWACA's digital library. The 315 studies reviewed cover the following four sub-themes: 1) teacher education and training and teaching practices; 2) learning assessment and quality of education; 3) curricular reforms and teaching methods; and 4) Non-formal education. These studies converge on a lack of initial and in-service teacher education, which makes it difficult for teachers to apply the pedagogical methods and curricula introduced by various reforms, and offer quality teaching. Twenty years after Maclure, teacher issues and teaching can be framed in the same terms. 


\section{RESUMEN}

\section{El magisterio y la enseñanza en la agenda de la Red occidental y central africana de investigación en educación (ROCARE): estado actual veinte años después de Maclure (1997)}

François Joseph AZOH, Escuela normal superior de Abidjan, Abidjan, Costa de marfil Affoué Philomène KOFFI, Universidad Felix Houphouët-Boigny, Abidjan, Costa de marfil Martial DEMBÉLÉ, Universidad de Montreal, Quebec, Canadá

Este artículo se inscribe en la prolongación de la síntesis realizada por Maclure en 1997 sobre la investigación en educación en África central y occidental. Así pues se trata de una revista de los trabajos de investigación sobre el tema del personal educativo y la enseñanza en los doce países francófonos miembros de la Red occidental y central africana de investigación en educación (ROCARE) a lo largo de los últimos veinte años, de 1997 a 2017. Esta revisión se basa en una bibliografía seleccionada en la biblioteca numérica de la ROCARE. Los 315 estudios identificados abordan los cuatro sub-temas siguientes: 1) formación del magisterio y practicas pedagógicas; 2 ) evaluación de aprendizajes y calidad de la educación; 3) reformas curriculares y métodos pedagógicos; 4) educación no formal. Esos estudios convergen en el déficit de la formación inicial y continua de los maestros y las maestras, lo que les impide aplicar los métodos pedagógicos y los curricula provenientes de las reformas y ofrecer una educación de calidad. Viente anos después de Maclure, el problema de los maestros y las maestras sigue vigente.

\section{CONTEXTE}

Créé en 1989 et conçu au départ comme un forum informel visant à regrouper les chercheuses et chercheurs en éducation de l'Afrique de l'Ouest et de l'Afrique centrale, le Réseau ouest et centre africain de recherche en éducation (ROCARE) est devenu l'une des principales institutions de recherche en éducation dans ces deux sous-régions. Ce réseau regroupe actuellement dix-sept pays, dont dix francophones, quatre anglophones, deux bilingues et un lusophone. Chaque pays constitue une coordination nationale, et l'ensemble est animé par un secrétariat exécutif. Les membres du réseau sont des universitaires, des enseignantes et enseignants ainsi que des étudiantes et étudiants intéressés par la recherche en éducation. Sa mission consiste à promouvoir l'expertise africaine en matière de recherche en éducation et une culture de la recherche afin d'améliorer les politiques et pratiques d'éducation dans les pays membres. 
Le mode d'élaboration du programme de recherche au sein du ROCARE est consensuel, en ce sens qu'il implique différentes entités du réseau. Plus précisément, le programme se construit selon deux démarches s'appuyant sur une concertation entre les coordinations nationales, le comité scientifique régional et le secrétariat exécutif. Dans la première démarche, de la base vers le sommet, les coordinations nationales proposent des thèmes de recherche et le secrétariat exécutif retient les plus récurrents et pertinents. Dans la seconde démarche, du sommet vers la base, le secrétariat exécutif propose des thèmes d'actualité qui constituent des enjeux pour l'éducation dans les pays membres du réseau.

La première démarche est celle qui est le plus fréquemment adoptée dans le réseau. Elle est valable de manière générale pour les études transnationales et le programme ROCARE des subventions pour la recherche en éducation ${ }^{1}$ destiné aux chercheuses et chercheurs débutants. La deuxième démarche est caractérisée par les recherches commanditées en général par les institutions partenaires en raison de leurs centres d'intérêt; c'est le cas de certaines études transnationales. Dans ce contexte, ces institutions proposent des thématiques de recherche dans le cadre d'un partenariat et financent les recherches. Ces dernières sont conduites par des équipes nationales composées de chercheurs débutants et expérimentés; leurs résultats sont par la suite transmis au secrétariat exécutif. Une équipe de chercheurs sélectionnés par ce secrétariat, en raison de leur expérience, présente ces résultats dans un rapport de synthèse.

En 1997, Maclure réalise un premier bilan de la recherche en éducation dans sept des dix pays membres du réseau, dont deux anglophones. Son objectif est d'effectuer la synthèse et de faire connaître les travaux de recherche réalisés dans ces pays, ceux-ci étant, pour la plupart, non publiés, méconnus et sous-utilisés par la communauté scientifique ainsi que les décideuses et décideurs. Ce bilan constitue une réponse aux inquiétudes des chercheuses et chercheurs africains, «isolés et sous financés et, par conséquent, incapables de produire de la recherche et de fournir les analyses nécessaires pour bien planifier et formuler de saines politiques éducatives» (Maclure, 1997, p. 1). Il répondait également aux préoccupations de plusieurs «organismes donateurs ayant beaucoup investi dans le développement de l'éducation» (p. 1) ainsi qu'au «besoin de développer les capacités analytiques des ministères africains et de promouvoir un milieu de recherche sain pour faciliter des réactions positives à la crise de l'éducation" (p. 2). Les études examinées

apportent un éclairage utile sur le personnel enseignant et l'enseignement, sur les facteurs influant sur l'apprentissage des élèves, sur les capacités d'innover en éducation, sur les caractéristiques de l'éducation traditionnelle et de l'éducation non formelle des adultes et sur la dynamique de

1. Mis en œuvre en 2002 grâce au financement du Centre de recherches pour le développement international (CRDI) du Canada, puis reconduit en 2006 grâce aux fonds du ministère des Affaires étrangères des Pays-Bas et de I'Union économique et monétaire ouest-africaine (UEMOA), le programme ROCARE des subventions pour la recherche en éducation a pour objectif d'assurer la relève scientifique par le renforcement des capacités de recherche des chercheuses et chercheurs débutants. 
l'engagement de la collectivité et du foyer envers l'école locale (Maclure, 1997, p. xiii).

Sur un total de $1056^{2}$ études répertoriées, Maclure a retenu 396 études pour la synthèse: 66 du Bénin, 84 du Burkina Faso, 45 du Cameroun, 34 du Ghana, 71 du Mali, 11 de la Sierra Leone et 85 du Togo.

Dans sa synthèse, Maclure (1997) consacre un chapitre au personnel enseignant et à l'enseignement, notre centre d'intérêt. Il y note que «l'ensemble de la recherche présente un tableau convaincant des enseignants et de l'enseignement qui exige une attention beaucoup plus grande qu'auparavant. Malheureusement, ce tableau est troublant» (p. 55-56). La plupart des études portant sur les pratiques pédagogiques font état de faiblesses et de déficiences majeures. Parmi les divers facteurs expliquant leur inefficacité, il y a la qualification du personnel enseignant, les difficiles conditions de vie et de travail, l'absence de supervision pédagogique et de soutien professionnel ainsi que les programmes et le matériel de travail vétustes et inadaptés.

En termes plus généraux, les méthodes d'enseignement ont été décrites de façons diverses comme ne réussissant pas à produire les niveaux prévus de connaissances chez les enfants, comme étant «anarchiques», et - ce qui est le plus important - comme contribuant directement à un rendement scolaire pauvre, à une faible estime de soi et à des aptitudes non convenables à une entrée éventuelle dans le monde du travail. (Maclure, 1997, p. 58)

Au terme de ce chapitre, Maclure dégage quatre perspectives de recherche: 1) la perception des enseignantes et enseignants par la communauté; 2) les attributs personnels et professionnels des enseignants; 3) l'éducation non formelle; 4) la recherche collaborative entre chercheurs et enseignants. La présente étude s'inscrit dans le prolongement de la synthèse de Maclure. Elle consiste en une revue des travaux de recherche réalisés sur le thème du personnel enseignant et de l'enseignement dans les douze pays francophones ${ }^{3}$ du ROCARE au cours des vingt dernières années (19972017). Nous nous limitons aux travaux réalisés par des chercheuses et chercheurs membres du réseau, qu'ils soient commandités ou soutenus par le secrétariat exécutif ou les coordinations nationales. Cette revue, quoique limitée, offre l'avantage de cerner la production scientifique du ROCARE sur ce thème.

2. Ce nombre concerne les productions nationales des pays membres, dont des travaux du ROCARE.

3. Il s'agit du Bénin, du Burkina Faso, du Cameroun, du Congo, de la Côte d'Ivoire, de la Guinée, du Mali, de la Mauritanie, du Niger, de la République centrafricaine, du Sénégal et du Togo. 


\section{LE PERSONNEL ENSEIGNANT ET LA QUALITÉ DE L'ÉDUCATION, UNE PROBLÉMATIQUE MONDIALE}

L'avènement de la société et de l'économie du savoir a imposé à tous les pays l'exigence de qualité en éducation, comme le souligne l'Organisation des Nations unies pour l'éducation, la science et la culture (UNESCO) :

Les débats récents sur la qualité de l'éducation [...] indiquent que la question de savoir ce qu'il faut entendre par qualité n'est pas tranchée. Cependant, une conclusion certaine est qu'une éducation de qualité (qu'elle soit dispensée à l'école ou sous d'autres formes d'apprentissage organisé) facilite l'acquisition des connaissances, des compétences et des attitudes qui ont une valeur intrinsèque et qui permettent aussi d'atteindre des objectifs humains importants (2004, p. 32 et 43).

Par ailleurs, bien que les opinions divergent, «trois principes tendent à être largement partagés [...] : la nécessité d'une plus grande pertinence, la nécessité d'une plus grande équité dans l'accès et les résultats et la nécessité d'un respect approprié des droits individuels» (UNESCO, 2004, p. 32). Au cœur des débats sur la qualité de l'éducation se trouve la question de la qualité du personnel enseignant et de l'enseignement. En effet, il existe un consensus sur le rôle crucial des enseignants dans l'offre d'une éducation de qualité et les résultats des élèves (Cusset, 2011; DarlingHammond, 2000; Dembélé et Gauthier, 2004; Hanushek, 2010; Kirk, Dembélé et Baxter, 2013; Institut de statistique de l'UNESCO, 2006; Lauwerier et Akkari, 2015; Lewin et Stuart, 2003; Organisation de coopération et de développement économiques, 2005; UNESCO, 2004). Ce consensus découle du constat de l'impact déterminant de l'enseignement dans les résultats d'apprentissage scolaire, solidement documenté au cours des cinquante dernières années.

L'enjeu est de taille en Afrique, où l'influence de l'enseignement, donc celle des enseignantes et enseignants, sur l'apprentissage semble plus grande que dans les pays développés. Si dans ces derniers «l'effet maître oscille généralement entre $5 \%$ et $15 \%$ de l'explication des acquisitions des élèves [...], la situation est tout autre dans [...] neuf pays [subsahariens] étudiés, où cet effet compte pour 27\%» (Bernard, Kouak Tiyab et Vianou, 2004, p. 33) ${ }^{4}$. Cette plus grande influence des enseignants est valable pour la plupart des pays africains marqués par la pauvreté, la ruralité, l'analphabétisme des parents, la rareté sinon l'absence de sources documentaires ainsi que la tradition de transmission orale. Dans ces conditions, l'enseignante ou l'enseignant demeure la source principale des apprentissages scolaires, et la performance des élèves dépend en bonne partie de la qualité de son enseignement. Dans de tels contextes, la question enseignante (y compris, entre autres, la formation, la motivation et le degré d'excellence des prestations du personnel enseignant) mérite que

4. Ces pays sont le Burkina Faso, le Cameroun, la Côte d'Ivoire, la Guinée, le Madagascar, le Mali, le Niger, le Sénégal et le Togo. 
l'on s'y attarde (Akyeampong, Lussier, Pryor et Westbrook, 2013; Bennell et Akyeampong, 2007; Lauwerier, 2016; Lewin et Stuart, 2003; Pôle de Dakar, 2009; Verspoor, 2005). C'est important d'étudier cette question d'autant que diverses évaluations régionales des apprentissages menées par le Programme d'analyse des systèmes éducatifs de la Conférence des ministres de l'Éducation des États et gouvernements de la Francophonie ${ }^{5}$ (PASEC) ont mis en lumière la faible qualité des acquis scolaires. L'évaluation conduite en 2008-2009 (PASEC, 2012) dans onze pays, dont six du ROCARE ${ }^{6}$, a donné les résultats suivants chez les élèves de cinquième année du primaire: 40,2\% et de 39,6\% respectivement en français et en mathématiques. Menée dans dix pays, dont huit du ROCARE7, l'évaluation de 2014 (PASEC, 2016) a montré que $70 \%$ et $50 \%$ des élèves de deuxième année du primaire se situent en dessous du seuil de réussite en français et en mathématiques respectivement. Elle a aussi montré que $60 \%$ des élèves se situent en dessous du seuil de réussite dans les mêmes matières en dernière année du primaire.

Au regard du rôle crucial du personnel enseignant dans les apprentissages des élèves et étant donné la mission et l'importance stratégique du ROCARE dans l'éducation en Afrique, il s'avère pertinent d'examiner la production scientifique de ce réseau relativement à la question enseignante, et ce, depuis la revue de Maclure (1997). En d'autres termes, qu'apprend-on de nouveau de cette production scientifique sur l'offre d'éducation, sa qualité, la formation des enseignantes et enseignants et leurs pratiques pédagogiques? Que peut-on en retenir pour éclairer le débat et les politiques dans les pays membres du réseau, en lien avec le quatrième objectif de développement durable de l'Organisation des Nations Unies (ONU) : «Assurer [d'ici 2030] l'accès de tous à une éducation de qualité, sur un pied d'égalité, et promouvoir les possibilités d'apprentissage tout au long de la vie» (ONU, 2015, section éducation de qualité)? C’est ce questionnement qui sous-tend la présente revue.

\section{DÉMARCHE MÉTHODOLOGIQUE}

Créée en 2008, la bibliothèque numérique du ROCARE ${ }^{8}$ a pour mission de faciliter l'accès à la production du réseau et à celle d'autres instances, résultant de travaux de recherche en éducation en Afrique de l'Ouest et en Afrique centrale. On y trouve

5. Créée en 1960, la Conférence des ministres de l'Éducation des États et gouvernements de la Francophonie (CONFEMEN) œuvre pour la promotion de l'éducation et de la formation professionnelle et technique auprès de ses 44 États et gouvernements membres. Elle leur offre un cadre d'échanges et de concertation sur les différents systèmes éducatifs et leur évolution. Le Programme d'analyse des systèmes éducatifs de la CONFEMEN (PASEC) vise à informer les membres sur l'évolution des performances des systèmes éducatifs afin de les aider à l'élaboration et au suivi de politiques éducatives. Les évaluations concernent les élèves en début et en fin de cycle primaire. Les disciplines évaluées sont la langue et les mathématiques.

6. Il s'agit du Bénin, du Cameroun, du Burkina Faso, du Congo, du Sénégal et de la Côte d'Ivoire.

7. Ces pays sont le Bénin, le Burkina Faso, le Cameroun, le Congo, la Côte d'Ivoire, le Niger, le Sénégal et le Togo.

8. Accessible en ligne (http://www.ernwaca.org/biblio/opac_css/index.php?lvl=index), cette bibliothèque réunit l'ensemble de la documentation du réseau. Elle permet d'avoir accès à de nombreux ouvrages et publications relatifs à la recherche en éducation en Afrique de l'0uest et en Afrique centrale. 
plusieurs types de documents: rapports d'études transnationales et nationales, rapports de recherche issus du programme de subventions qui s'adresse aux chercheuses et chercheurs débutants, articles de revue et travaux de consultance; il s'agit de documents produits depuis 1989 et classés en 25 catégories. Cette bibliothèque est la principale source du corpus documentaire constitué pour la présente revue.

Nous avons procédé à la recension des études réalisées au cours des vingt dernières années dans chacune des 25 catégories en utilisant les mots-clés suivants: enseignement, enseignant, pratiques pédagogiques et formation des enseignants. Nous avons sélectionné l'ensemble des études portant sur notre sujet de recherche à partir des titres ou des résumés, pour en extraire celles réalisées dans les pays francophones. Pour chaque étude retenue, nous avons créé une fiche de lecture, qui précise entre autres les objectifs et les principaux résultats de l'étude. Nous avons ensuite classé les études par sous-thème et comparé les résultats par similarité et opposition.

\section{RÉSULTATS}

De 1997 à 2017, la production du ROCARE s'élève à 507 études: 405 d'entre elles $(80 \%)$ traitent de la question enseignante et parmi ces dernières, 315 émanent des pays francophones, ce qui représente presque $78 \%$ des études sur le sujet. C'est dire que cette question est au centre des préoccupations des chercheuses et chercheurs membres du réseau. Comme le montre la figure 1, les travaux réalisés dans le cadre du programme de subventions destiné aux chercheurs débutants représentent $45,69 \%$ des 315 études retenues, ce qui témoigne de la volonté du réseau d'assurer la relève scientifique en son sein.

Figure 1. Répartition des études réalisées par le ROCARE de 1997 à 2017 par catégorie

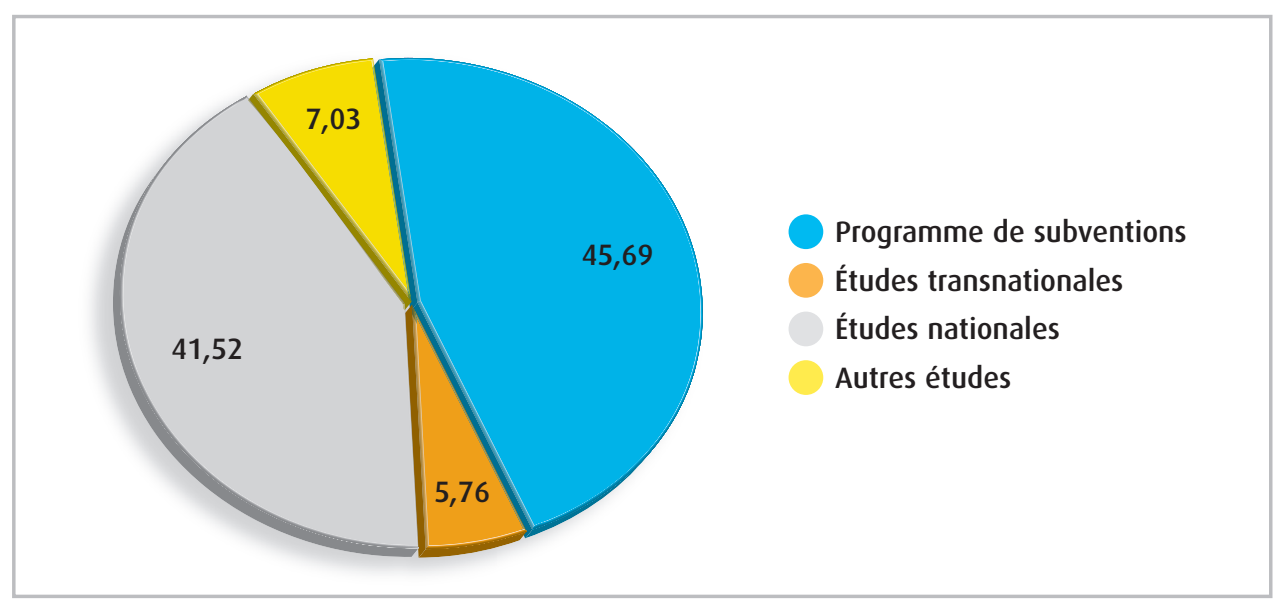


Ces études couvrent quatre sous-thèmes prépondérants: 1) formation du personnel enseignant et pratiques pédagogiques $(39,87 \%) ; 2$ ) évaluation des apprentissages et qualité de l'éducation $(24,68 \%)$; 3 ) réformes curriculaires et méthodes pédagogiques $(17,78 \%) ; 4)$ éducation non formelle (16,45\%) (voir le tableau 1). C'est autour de ces sous-thèmes que nous présentons les résultats de notre revue. La contrainte d'espace nous a amenés à présenter seulement les constats généraux qui ressortent des études recensées.

Tableau 1. Répartition des études du ROCARE selon les sous-thèmes et la couverture géographique

\begin{tabular}{|c|c|c|c|}
\hline Sous-thème & $\begin{array}{l}\text { Nombre } \\
\text { d'études }\end{array}$ & $\begin{array}{l}\text { Proportion } \\
(\%)\end{array}$ & Pays couverts \\
\hline $\begin{array}{l}\text { Formation du personnel } \\
\text { enseignant et pratiques } \\
\text { pédagogiques }\end{array}$ & 125 & 39,87 & $\begin{array}{l}\text { Bénin, Burkina Faso, Cameroun, Côte d'Ivoire, } \\
\text { Guinée, Mali, Mauritanie, République centrafricaine, } \\
\text { Sénégal et Togo. }\end{array}$ \\
\hline $\begin{array}{l}\text { Évaluation des apprentissages } \\
\text { et qualité de l'éducation }\end{array}$ & 78 & 24,68 & $\begin{array}{l}\text { Bénin, Burkina Faso, Cameroun, Côte d'Ivoire, } \\
\text { Guinée, Mali, Mauritanie, Niger, Sénégal et Togo. }\end{array}$ \\
\hline $\begin{array}{l}\text { Réformes curriculaires et } \\
\text { méthodes pédagogiques }\end{array}$ & 56 & 17,78 & $\begin{array}{l}\text { Bénin, Cameroun, Congo, Côte d’Ivoire, Mali, Niger, } \\
\text { République centrafricaine et Sénégal. }\end{array}$ \\
\hline Éducation non formelle & 52 & 16,45 & $\begin{array}{l}\text { Bénin, Burkina Faso, Côte d’Ivoire, Guinée, Mali, } \\
\text { Mauritanie, Niger et Sénégal. }\end{array}$ \\
\hline Autres & 4 & 1,22 & Guinée et Burkina Faso. \\
\hline Total & 315 & 100 & \\
\hline
\end{tabular}

\section{Formation du personnel enseignant et pratiques pédagogiques}

Les recherches en lien avec ce premier sous-thème traitent de la formation initiale et continue (Fomba et Diarra, 2003; Tchagnaou et Baoutou, 2016), des pratiques de classe (Camara, Mansaré, Barry et Sy, 2009; Ouattara-Goïta, N'dédé et Aya, 2014; Walet, Bocoum, Bamba et Faye, 2010) et des compétences du personnel enseignant (Ouattara, N'dédé et Aya, 2009; Rabiou et Sani, 2006; Sène, 2008). Ces études concluent, pour la plupart, que les enseignantes et enseignants ne sont pas suffisamment formés pour appliquer les méthodes pédagogiques issues des réformes, telles que la formation par compétences et l'approche par compétences. Par conséquent, ces méthodes sont partiellement ou mal appliquées dans les classes. Ouattara et al. (2009) constatent que les insuffisances de formation des enseignants conduisent à des confusions entre la formation par compétences et la pédagogie par objectifs, l'approche précédemment en vigueur au primaire en Côte d'Ivoire. Les études mettent en cause la capacité des décideuses et décideurs à choisir des méthodes pédagogiques et à planifier leur implantation et leur appropriation. En effet, ces méthodes ne connaissent généralement pas de phase d'expérimentation et de maturation avant leur généralisation, ce qui conduit indubitablement à des difficultés d'application. 
Toujours en lien avec le sous-thème de la formation et des pratiques pédagogiques, la question de l'intégration pédagogique des technologies de l'information et de la communication (TIC) a suscité de l'intérêt parmi les chercheuses et chercheurs. L'Agenda panafricain de recherche sur l'intégration pédagogique des TIC (PanAf) ${ }^{9}$, qui a démarré en 2008, a été le principal véhicule de cet intérêt. Les chercheurs de sept pays membres du ROCARE, dont six francophones ${ }^{10}$, ont réalisé des travaux dans le cadre d'une étude transnationale dont les résultats conduisent au même constat: il y a une insuffisance de formation chez le personnel enseignant quant à l'usage pédagogique des TIC (Azonhe, Adjibodou et Akouété-Hounsinou, 2008; Maiga, 2010; Mbangwana, 2008; Mian, 2016; N'dédé, 2012; Tsigbe, 2010) ${ }^{11}$. Azonhe et al. (2008) constatent au Bénin que l'usage pédagogique des TIC par les enseignantes et enseignants repose principalement sur le recours constant de leurs élèves à Internet, ce qui les oblige à s'adapter à l'usage de ces technologies qui s'invitent de plus en plus dans l'espace scolaire.

Les recommandations issues de l'ensemble de ces travaux portent sur la formation continue, l'installation d'un dispositif de suivi des enseignants dans les écoles, l'autoformation par l'usage des TIC. Elles mettent l'accent sur l'accès à des documents pédagogiques et l'évaluation des réformes.

\section{Évaluation des apprentissages et qualité de l'éducation}

La qualité de l'éducation est un sous-thème important et transversal dans la recherche du ROCARE. En 2002, il a fait l'objet d'une étude transnationale regroupant sept pays, dont cinq francophones ${ }^{12}$. Ce sous-thème est également abordé dans le programme ROCARE des subventions pour la recherche en éducation sous les deux thèmes suivants: «Réforme des curricula pour/et l'amélioration de la qualité en éducation", en 2009, et "Qualité de l'éducation en Afrique: définition, indicateurs et pratiques", en 2010 .

Des études concernent l'impact de l'organisation scolaire sur la qualité de l'éducation (Koffi, Koffi et Ouohi, 2010; Koffi, N'cho et N'guessan, 2010), l'effet de la participation communautaire sur la qualité de l'éducation (Salami et Kpamegan, 1997; Sangaré et Diarra, 1997), les réformes éducatives et la qualité de l'éducation (Bocoum, Thiero, Dia et Ag, 2009; Montcho, Attenoukon, Sossa, Kenoukon et Kpenonhou, 2011;

9. L'Agenda panafricain de recherche sur l'intégration pédagogique des TIC, d'une durée de quatre ans, soutenu par le CRDI et I'Université de Montréal, avait pour objectif de "mieux comprendre comment l'intégration des TIC peut améliorer la qualité des enseignements et des apprentissages en Afrique » (Karsenti, Collin et Harper-Merret, 2012). Ce projet s'est déroulé en deux phases, de 2007 à 2011, et a impliqué des équipes de recherche issues de treize pays africains.

10. Ces pays sont le Cameroun, le Congo, la Côte d'Ivoire, le Mali, la République centrafricaine et le Sénégal.

11. Cette étude a permis de mettre en place un observatoire des TIC dans le domaine de l'éducation en Afrique. Accessible en ligne (http://www.observatoiretic.org/default/use?locale=fr), cet observatoire est une base de données libre d'accès pour la recherche sur l'intégration pédagogique des TIC.

12. Il s'agit du Bénin, du Cameroun, de la Côte d'Ivoire, du Mali et du Togo. 
Rabiou, Boube et Moussa, 2010). Elles concluent que la qualité de l'éducation dans les établissements scolaires est faible en raison d'une maîtrise insuffisante des réformes éducatives par le personnel enseignant et des difficiles conditions de travail. En ce qui concerne la participation communautaire, les études montrent une implication financière, matérielle et humaine des communautés dans l'amélioration de l'offre d'éducation à travers la construction d'infrastructures scolaires et la fourniture d'équipements. Toutefois, ces efforts ne permettent pas d'obtenir une éducation de qualité à cause de l'insuffisance de matériel didactique, de formation et de supervision des enseignants.

La faible qualité de l'éducation relève aussi des procédures d'évaluation des apprentissages (Konaté, Damba et Sidibé, 2007; Konaté, 2016; Rabiou et Sani, 2006; Tchagnaou et Baoutou, 2016). Les résultats des études recensées révèlent de nombreuses lacunes dans les procédures d'évaluation des apprentissages, notamment la non-conformité du mode d'évaluation à l'approche pédagogique préconisée ainsi que l'irrégularité des évaluations. Konaté (2016) souligne que l'attention est plus orientée sur les notes obtenues par l'élève lors des évaluations que sur la compétence à acquérir, ce qui est contraire aux principes de l'approche par compétences qui est censée être pratiquée dans les écoles maliennes. L'ensemble des études font état de difficultés relevant de l'indisponibilité ou de la vétusté du matériel d'évaluation et du ratio élèves-enseignant élevé.

Les études recommandent la mise en place de structures chargées de l'évaluation des apprentissages et de l'enseignement, notamment pour l'élaboration d'instruments d'évaluation. Elles insistent sur la formation du personnel enseignant aux méthodes pédagogiques et en docimologie.

\section{Réformes curriculaires et méthodes pédagogiques}

Les nouvelles exigences éducatives liées aux problèmes sociétaux actuels ont conduit à l'intégration de nouvelles matières aux programmes scolaires (UNESCO, 2012), telles que l'éducation à la citoyenneté (Azoh, 2011; Kanon, Bakayoko, Inanan et Moussa, 2008), l'éducation à l'environnement (Dahiratou, Bassirou, Moctar et Garba, 2009; Djané, Konaté et Kouassi, 2009; Rabiou, 2014) et l'éducation à l'entrepreneuriat (Bénié, 2012; Sénou, Soglo, Quenum, et Okan, 2010). L'enseignement des langues nationales et dans ces langues (Bousso, Samba, Faye et Claverane, 2009; Samba et Bousso, 2014) est une exigence supplémentaire intégrée par plusieurs pays.

L'ensemble de ces études montre la nécessité d'instaurer ces nouveaux programmes scolaires pour améliorer la qualité et la pertinence de l'offre d'éducation. Leur intégration a pour finalité de conformer les systèmes éducatifs aux nouveaux enjeux sociétaux dans l'éducation. Il s'agit d'amener les élèves à acquérir de nouvelles connaissances et à développer des habiletés pour améliorer leurs conditions de vie. 
Les études évaluent les contenus des programmes et leur appropriation par les élèves. Les résultats indiquent des insuffisances dans les connaissances des élèves qui découlent principalement de déficits dans la formation initiale et continue du personnel enseignant. Dahiratou et al. (2009) expliquent que la qualité des cours en éducation à l'environnement dans les établissements scolaires nigériens est compromise par une appropriation et une planification insuffisante des contenus éducatifs. Les recommandations de ces chercheuses et chercheurs visent principalement la formation du personnel enseignant et celle de ses formateurs et formatrices. Ils proposent l'intégration des nouveaux programmes scolaires à la formation initiale des enseignantes et enseignants. Ils recommandent par ailleurs un volet pratique dans l'enseignement de ces nouveaux programmes en impliquant davantage les associations de parents d'élèves.

\section{Éducation non formelle}

L'éducation non formelle est une réponse complémentaire à la demande d'éducation formelle et se présente sous plusieurs formes: classes passerelles ${ }^{13}$, écoles confessionnelles islamiques, médersas, écoles communautaires.

Des études transnationales ont porté sur ce sous-thème en lien avec le VIH et le sida (Azoh, 2005; Azoh et Yaro, 2007), avec les médersas et les autres centres d'éducation coranique (Laouali et Benett, 2007) ${ }^{14}$. Il a également fait l'objet de travaux de recherche dans le cadre du programme ROCARE des subventions pour la recherche en éducation sur deux thèmes, soit «L'éducation non formelle: méthodes traditionnelles de l'enseignement du Coran», en 2009, et "L'éducation formelle et l'éducation non formelle: inclusion et qualité», en 2016. À l'échelle nationale, les recherches ont porté sur les écoles communautaires et confessionnelles islamiques (Kakaï, Legba, Gansa et Tossou, 2009; Silué et N'djoré, 2016; Tounkara, Fomba et Fané, 2001), sur l'alphabétisation (Sy et Traoré, 1997) ainsi que sur la santé du personnel enseignant du sous-secteur non formel (Macalou et Konaté, 2006).

Les résultats de ces recherches indiquent un manque de formation chez le personnel enseignant de ce sous-secteur. Macalou et Konaté (2006) estiment qu'il est important de combler le manque de connaissances des élèves et du personnel enseignant sur le VIH et le sida. En Côte d'Ivoire, des études (N'Guessan et N'djoré, 2016; Silué et N'djoré, 2016) ont évalué les conditions favorables à l'intégration des écoles confessionnelles islamiques au système éducatif formel. Dans le cadre de ce projet, des conseillères et conseillers pédagogiques apportent un encadrement aux enseignantes et enseignants,

13. La classe passerelle est une forme alternative d'organisation de l'enseignement qui offre une deuxième chance aux enfants de 8 à 12 ans, jamais inscrits ou exclus du système d'éducation formel.

14. Cette étude est disponible uniquement en version anglaise alors qu'elle concerne quatre pays, dont trois francophones (Mali, Niger et Sénégal). 
mais cet encadrement ne semble pas suffisant pour améliorer leurs aptitudes à enseigner.

Quant à Tounkara et al. (2001), ils montrent que la qualité de l'enseignement offert dans les écoles communautaires gérées par des organisations non gouvernementales est supérieure ou comparable à celle des écoles publiques. Les taux de réussite des élèves de ces écoles communautaires en français et en mathématiques sont supérieurs à ceux des élèves des écoles publiques. Cette différence repose sur la formation initiale, la disponibilité du matériel de travail, l'encadrement et la supervision: $100 \%$ des enseignantes et enseignants des écoles communautaires disposent du document du programme officiel et $84 \%$ possèdent le guide du maître contre respectivement $57 \%$ et $43 \%$ du personnel enseignant des écoles publiques.

Ces auteurs encouragent le développement de l'éducation non formelle par le développement des compétences pédagogiques du personnel enseignant et des compétences administratives des directions d'école pour une gestion efficace des programmes.

\section{DISCUSSION}

Le but de cette revue était de faire un état des lieux de la recherche en éducation réalisée par les chercheuses et chercheurs francophones du ROCARE ces vingt dernières années, après la synthèse de Maclure en 1997. Les résultats montrent que la production du ROCARE est abondante, ce qui traduit une certaine vitalité du réseau en matière de recherche. Les études recensées abordent l'éducation dans plusieurs contextes nationaux et selon différents angles, permettant ainsi d'enrichir les connaissances. La variété et la richesse des travaux permettent d'informer le personnel enseignant ainsi que les décideuses et décideurs sur les problèmes d'éducation et les politiques pour la prise de décisions.

La formation du personnel enseignant et les pratiques pédagogiques ont fait l'objet de nombreuses études qui, pour la plupart, mettent en avant la formation insuffisante de ce personnel et son incapacité à mettre en œuvre les nouvelles approches pédagogiques. Cette question demeure persistante depuis la revue de Maclure (1997), qui révélait des lacunes dans la formation des enseignantes et enseignants, se traduisant par le manque de compétences de ceux-ci dans les salles de classe, les méthodes d'évaluation des apprentissages inadaptées et lacunaires et, par ricochet, le faible rendement scolaire des élèves. Les ratios élèves-enseignant et le manque de matériel pédagogique sont d'autres facteurs explicatifs de cette situation. L'insuffisance de formation du personnel enseignant a été relevée par d'autres études (Lauwerier et Akkari, 2015; Lauwerier, 2016), qui affirment que dans de nombreux pays africains, les réformes n'influencent pas les pratiques pédagogiques des enseignantes et enseignants. La durée de la formation est trop courte et «même si les 
enseignants connaissent bien le contenu du curriculum, ils n'ont pas tous les outils pédagogiques nécessaires pour l'enseigner» (Lauwerier et Akkari, 2015, p. 4).

L'éducation non formelle présente elle aussi des faiblesses qualitatives attribuables aux insuffisances de formation et d'encadrement pédagogique des enseignants. Depuis Maclure, les recherches ont dénoncé la faible qualité de l'offre d'éducation dans ce sous-secteur et ont suggéré un partenariat entre cette forme d'éducation et l'éducation formelle en vue de son amélioration. La présente revue note une mutation de cette forme d'éducation par son intégration au système formel.

Les recherches se sont également intéressées aux réformes curriculaires. À ce chapitre, elles ont montré l'importance de l'intégration de nouveaux programmes scolaires en vue d'améliorer et d'adapter l'offre éducative. Les résultats indiquent des lacunes dans les connaissances du personnel enseignant et des élèves. Ces réformes, implantées pour corriger la monotonie et l'impertinence des programmes (Maclure, 1997), n'ont donc pas produit les effets escomptés.

Les problèmes d'offre éducative auxquels font face les systèmes éducatifs des pays couverts par les études recensées mettent en doute la pertinence de certaines réformes et démontrent la nécessité de les adapter à leurs réalités. Il y va de la réalisation de l'objectif 4 de développement durable de l'Organisation des Nations Unies qui, rappelons-le, est d'«assurer [d'ici 2030] l'accès de tous à une éducation de qualité, sur un pied d'égalité, et promouvoir les possibilités d'apprentissage tout au long de la vie» (ONU, 2015, section éducation de qualité).

\section{CONCLUSION}

Les études réalisées sur la question enseignante et l'enseignement au cours des vingt dernières années dans les pays francophones membres du ROCARE portent aussi bien sur des thèmes traités par Maclure (1997) que sur de nouveaux thèmes. Des efforts ont été faits pour améliorer l'offre éducative, mais la qualité de l'éducation demeure faible en raison du manque de formation du personnel enseignant. Vingt ans après Maclure, la question enseignante et l'enseignement se présentent dans les mêmes termes.

La recherche sur la formation du personnel enseignant et l'enseignement n'est malheureusement pas suffisamment connue et exploitée en raison de l'absence de collaboration de la communauté scientifique avec les gouvernements nationaux et les autres partenaires de l'éducation. Cela explique aussi la faible emprise de ces recherches sur les politiques éducatives des États membres. Or le développement d'une expertise locale en matière de recherche doit permettre d'éclairer les décisions politiques. Cela passe nécessairement par le développement des compétences et de l'autonomie des chercheuses et chercheurs. 
Dans le cadre de l'Agenda panafricain de recherche sur l'intégration pédagogique des TIC (PanAf), les recherches ont permis de lancer un dialogue politique pour inciter les décideuses et décideurs à prendre des mesures pour intégrer ces technologies dans l'enseignement. Initié par un pays du Nord, ce programme est le fruit d'une collaboration tripartite entre le Centre de recherches pour le développement international du Canada, pour le soutien financier, l'Université de Montréal, pour le soutien scientifique, et le ROCARE, pour la réalisation des travaux de recherche et l'animation du plaidoyer. Cette approche est à promouvoir en raison des renforcements scientifiques qu'elle a apportés aux équipes de recherche.

Toutefois, le peu de recherches menées sur l'intégration pédagogique des TIC depuis la fin du programme PanAf soulève la question de la dépendance financière du ROCARE vis-à-vis de ses partenaires internationaux. L'orientation des sujets de recherche imposée par les institutions commanditaires provoque une forte dépendance des équipes de recherche vis-à-vis de ces institutions et ne favorise pas leur autonomie intellectuelle. Les financements nationaux, actuellement inexistants, ne permettent pas aux chercheuses et chercheurs de gagner en autonomie dans les choix des sujets de recherche, comme l'indiquait Maclure (1997). Par conséquent, une politique de mobilisation des ressources auprès des États et du secteur privé devrait être mise en place. Au regard de l'exigence de qualité en éducation, des constats problématiques faits à ce sujet dans les pays membres du ROCARE et des éclairages que les chercheurs peuvent apporter en la matière, les gouvernements de ces pays ont tout intérêt à investir davantage dans la recherche en éducation, un secteur névralgique du développement national.

Une des limites de la présente revue est qu'elle ne s'est pas penchée sur les aspects méthodologiques des travaux recensés. La prise en compte de cet aspect devrait permettre une analyse de la qualité de la recherche produite par le ROCARE. Cela est d'autant important que presque la moitié des travaux en question ont été réalisés dans le cadre du programme de subventions destiné aux chercheuses et chercheurs débutants.

Enfin, une question d'actualité qui n'a pas été abordée dans les recherches est celle de l'attrition du personnel enseignant. La recherche nationale et transnationale au sein du réseau devrait s'y intéresser au regard du nombre important d'enseignantes et enseignants qui quittent le métier pour des raisons autres que la retraite en Afrique de l'Ouest et en Afrique centrale ces dernières années. 


\section{Références bibliographiques}

AKYEAMPONG, K., LUSSIER, K., PRYOR, J. et WESTBROOK, J. (2013). Improving teaching and learning of basic maths and reading in Africa: Does teacher preparation count? International Journal of Educational Development, 33(3), 272-282.

AZOH, F. J. (2005). La contribution de l'éducation non formelle à la prévention du VIH ISIDA. Hambourg, Allemagne: Unesco Institute for Education.

AZOH, F. J. (2011). Attitudes et représentations sociales face aux pratiques corruptives ou comment éduquer à la citoyenneté? Revue africaine de recherche en éducation, 3, 1-8.

AZOH, F. J. et YARO, Y. (2007). Perspectives transnationales sur l'éducation de base en Afrique centrale et de l'ouest: accès, qualité et participation (rapport de recherche). Repéré à www.rocare.org

AZONHE, T.H., ADJIBODOU, A., et AKOUÉTÉ-HOUNSINOU, F. (2008). Comment intégrer les TIC dans les écoles béninoises si les enseignantes restent en marge? Dans K. Touré, T. M. S. Tchombe et T. Karsenti (dir.), ICT and changing mindsets in education (p. 65-75). Bamenda, Cameroun: Langaa.

BÉNIÉ, A. H. (2012). Attentes relatives à la formation initiale et choix professionnel chez les étudiants. African Educational Development Issues, 4, 37-60.

BENNELL, P. et AKYEAMPONG, K. (2007). Teacher motivation in Sub-Saharan Africa and South Asia. Londres, Royaume Uni: Central Research Department of the Department for International Development.

BERNARD, J.-M., KOUAK TIYAB, B. et VIANOU, K. (2004). Profils enseignants et qualité de l'éducation primaire en Afrique subsaharienne francophone: bilan et perspectives de dix années de recherche du PASEC. Dakar, Sénégal: CONFEMEN.

BOCOUM, I., THIERO, F., DIA, M. et AG, A. A. (2009). Approche par compétences et qualité de l'éducation: cas des écoles du Centre d'animation pédagogique de Kalabancoro (région de Koulikoro) (rapport de recherche). Bamako, Mali: ROCARE.

BOUSSO, Y. D. A., SAMBA, I., FAYE, P. E. et CLAVERANE, C. (2009). L'introduction des langues nationales dans le système éducatif formel entre médium de communication et outils d'apprentissage scolaire (rapport de recherche). Bamako, Mali: ROCARE. 
CAMARA, M., MANSARE, D., BARRY, A. B. et SY, N. (2009). Du curriculum formel au curriculum enseigné: comment les maîtres contractuels comprennent et mettent en ouvre le programme rénové de l'enseignement élémentaire? (rapport de recherche). Bamako, Mali: ROCARE.

CUSSET, J.-Y. (2011). Que disent les recherches sur l'«effet enseignant»? Questions sociales. La note d'analyse. 232. Repéré à http://archives.strategie.gouv.fr/cas/system /files/na-qsociales-232.pdf

DAHIRATOU, M., BASSIROU A., MOCTAR, A. M. et GARBA, M. (2009). L'éducation à l'environnement dans les systèmes éducatifs nigériens (rapport de recherche). Bamako, Mali: ROCARE. Repéré à http://www.ernwaca.org/biblio/opac_css/index. php?lvl=index

DARLING-HAMMOND, L. (2000). Teacher quality and student achievement: A review of state policy evidence. Education Policy Analysis Archives, 8(1). Repéré à http:// epaa.asu.edu/epaa/v8nl

DEMBÉLÉ, M. et GAUTHIER, C. (2004). Qualité de l'enseignement et qualité de l'éducation: revue des résultats de recherche. Document de référence préparé pour le Rapport mondial de suivi sur l'Éducation pour tous 2005.

DJANÉ, K. A., KONATÉ, A. et KOUASSI, F. C. (2009). Éducation environnementale à l'école primaire et adoption du comportement environnemental par l'élève (rapport de recherche). Bamako, Mali: ROCARE. Repéré à http://www.ernwaca. org/biblio/opac_css/index.php?lvl=index

FOMBA, C.O. et DIARRA, M. F. (2003). Évaluation de l'impact des formations des contractuels de l'éducation sur leurs pratiques de classes. Rapport de recherche. ROCARÉ / Unicef, Bamako. Repéré à http://www.ernwaca.org/biblio/opac_css/ index.php?lvl=index

HANUSHEK, E. (2010). The difference is great teachers. In K. Weber (dir.), Waiting for superman: How can we save America's failing public schools (p. 81-100). New York, NY: Public Affairs.

IINSTITUT DE STATISTIQUE DE L'UNESCO. (2006). Les enseignants et la qualité de l'éducation: suivi des besoins mondiaux d'ici 2015. Montréal, Québec: UNESCOISU.

KAKAÏ, G., LEGBA, R., GANSA, B. et TOSSOU, R. (2009). Le métier d'enseignant communautaire: Profil de l'éducateur et impact sur le système éducatif béninois (Rapport de recherche). Bamako, MALI: ROCARE. Repéré à http://www.ernwaca. org/biblio/opac_css/index.php ?lvl=index 
KANON, G. L., BAKAYOKO, A. V., INANAN, K. G. et MOUSSA, M. (2008). Paix et éducation à la citoyenneté. La contribution des acteurs (rapport de recherche). Bamako, Mali: ROCARE. Repéré à http://www.ROCARE.org/grants/2008/PaixEducation-Citoyennete.pdf

KARSENTI, T., COLLIN, S. et HARPER-MERRET, T. (2012). Intégration pédagogique des TIC: succès et défis de 100+ écoles africaines. Ottawa. CRDI. Repéré à http://www.observartoiretic.org/documents/2011-11_livre_fr.pdf

KIRK, J., DEMBÉLÉ, M. et BAXTER, S. (Eds.) (2013). More and betters teachers for quality education for all: Identity and motivation, systems and support. Brighton, Angleterre: Collaborative Works. Repéré à http://moreandbetterteachers.wordpress. com/

KOFFI, A. M.-C., N'CHO, L. et N'GUESSAN, H. (2010). Politique d'efficacité interne et qualité de l'éducation dans le secondaire général: cas des établissements publics d'Abidjan et de Yamoussoukro (rapport de recherche). Bamako, Mali: ROCARE. Repéré à http://www.ernwaca.org/biblio/opac_css/index.php?lvl=index

KOFFI, A. P., KOFFI, A. H. et OUOHI, C. (2010). Climat scolaire et performance des établissements publics d'enseignement secondaire d'Abidjan (rapport de recherche). Bamako, Mali: ROCARE. Repéré à http://www.ernwaca.org/biblio/ opac_css/index.php?lvl=index

KONATÉ, B. (2016). Évaluation des apprentissages scolaires au mali: nécessité de changement de paradigme. Revue de vulgarisation du ROCARE, 1, 34-37.

KONATÉ, B., DAMBA, K. et SIDIBÉ, K. (2007). Évaluation des apprentissages à l'Université de Bamako: forces et faiblesses. Revue africaine de recherche en éducation, 2, 11-17.

LAOUALI, M. M. et BENETT, Y. (2007). A synthesis of studies of Madrassas and other Quranic Schooling Centres in Gambia, Mali, Niger and Senegal (rapport de recherche). Bamako, Mali: ROCARE. Repéré à http://www.ernwaca.org/biblio/ opac_css/index.php?lvl=index

LAUWERIER, T. (2016). La contribution des enseignants à la pertinence de l'éducation de base en Afrique de l'Ouest: le cas du Sénégal, McGill Journal of Education, 51(2), 787-805.

LAUWERIER, T. et AKKARI, A. (2015). Les enseignants et la qualité de l'éducation de base en Afrique subsaharienne. Recherche et prospective en éducation, UNESCO, Paris [Réflexions thématiques, No 11]. 
LEWIN, K. M. et STUART, J. S. (2003). Researching teacher education: new perspectives on practice, performance and policy-multi-site teacher education research project (MUSTER) synthesis report. Londres, Angleterre: Department for International Development (DFID).

MACALOU, C., D. et KONATE, S. (2006). Lutte contre la stigmatisation et la discrimination des personnes vivant avec le VIH/SIDA au moyen de l'éducation non formelle dans les communautés de Koulokoro et de Banamba en république du Mali. (Rapport de recherche). Bamako, Mali: ROCARE. Repéré à http://www. ernwaca.org/smgrt2006-ml-vih.pdf

MACLURE, R. (1997). Négligée et sous-estimée: la recherche en éducation en Afrique centrale et Afrique occidentale. Une synthèse d'études nationales du ROCARÉ. Bamako, Mali: ROCARE.

MAIGA, M. (2010). Obstacles et défis à l'intégration pédagogique des TIC dans l'éducation en Afrique. Communication au Programme Panaf-e-learning Africa, 26-28 mai 2010, FOAD/Lusaka. Repéré à http://www.ernwaca.org/biblio/opac_css/ index.php ?|vl=index

MBANGWANA, M. (2008). Introduction of ICT in Schools and Classrooms in Cameroon. Dans K. Touré, T. M. S. Tchombe et T. Karsenti (dir.), ICT and changing mindsets in education (p. 113-124). Bamenda, Cameroun: Langaa.

MIAN, B. S., A. (2016). Les usages des TIC pour la formation continue des enseignants en Côte d'Ivoire: cas de l'Université de vacances de l'ENS d'Abidjan, Revue de vulgarisation ROCARE, 1, 25-28.

MONTCHO-A., E., ATTENOUKON, S., SOSSA, L., KENOUKON, C. et KPENONHOU, C. (2011). La qualité des formations délocalisées dans l'enseignement supérieur au Bénin. African Educational Development Issues, 3, 11-26.

N'DÉDÉ, B. F. (2012). Usages pédagogiques des technologies de l'information et de la communication (TIC) par les élèves du secondaire en Côte d'Ivoire. African Educational Development Issues, 4, 210-220.

N'GUESSAN, G. K. D. et N'DJORE, Y. A. B. (2016). Scolarisation en Côte d'Ivoire: l'expérience des écoles confessionnelles islamiques intégrées. Revue de vulgarisation ROCARE, 1, 42-45.

ORGANISATION DE COOPÉRATION ET DE DÉVELOPPEMENT ÉCONOMIQUES (OCDE). (2005). Le rôle crucial des enseignants. Attirer, former et retenir des enseignants de qualité. Paris, France: auteur. 
ORGANISATION DES NATIONS UNIES (ONU). (2015). Objectifs du développement durable. 17 objectifs pour transformer notre monde. Repéré à http://www.un.org/ sustainabledevelopment/fr/objectifs-de-developpement-durable/

OUATTARA, K., N'DÉDÉ, B. F. et AYA, A. (2009). La formation par compétences dans l'enseignement primaire en Côte d'Ivoire: réalité et défis (rapport de recherche), Bamako, Mali: ROCARE. Repéré à http://www.ernwaca.org/biblio/opac_css/index. php?lvl=index

OUATTARA-GOÏTA, K. I., N’DÉDÉ, B. F. et AYA, A. (2014). Perceptions et difficultés liées à la Formation Par Compétences (FPC) dans les écoles primaires de Côte d'Ivoire. African Educational Development Issues, 6, 34-50.

PROGRAMME D'ANALYSE DES SYSTĖMES ÉDUCATION DE LA CONFEMEN (2012). Synthèse des résultats PASEC VII, VIII et IX. Dakar: auteur. Repéré à http://www. pasec.confemen.org/wp-content/uploads/2015/07/31-Synthese_PASEC_VII-VIIIIX_final.pdf

PROGRAMME D'ANALYSE DES SYSTÈMES ÉDUCATION DE LA CONFEMEN (2016). Performances des systèmes éducatifs en Afrique subsaharienne francophone. Compétences et facteurs de réussite au primaire. Dakar: auteur.

PÔLE DE DAKAR. (2009). La scolarisation primaire universelle en Afrique: le défi enseignant. Dakar, Sénégal: UNESCO-BREDA.

RABIOU, R. (2014). L'éducation à la citoyenneté par l'école: une nécessité au Niger! African Educational Development Issues, 5, 55-70.

RABIOU, R., BOUBE, M. et MOUSSA L. M. (2010). Contractualisation et qualité de l'enseignement de base au Niger. African Educational Development Issues, 1, 6984.

RABIOU, R. et SANI, B. M. (2006). Impact de la contractualisation sur la qualité de l'enseignement de base 1 au Niger (rapport de recherche), Bamako, Mali: ROCARE. Repéré à http://www.ROCARÉ.org/smgrt2006-niger-employment.pdf

RÉSESAU OUEST ET CENTRE AFRICAIN DE RECHERCHE EN ÉDUCATION (ROCARE). (2012). Programme ROCARE des subventions pour la recherche en éducation. Nouvelles du ROCARE, édition spéciale Subventions ROCARE pour la recherche en éducation. Bamako, Mali: Auteur. Repéré à http://www.ernwaca.org/ biblio/opac_css/index.php?lvl=index

SALAMI, N., D. et KPAMEGAN, G. (1997). Effets de la participation communautaire sur l'accès et la qualité de l'éducation de base: expériences du Bénin (rapport de recherche). Bamako, Mali: ROCARE. 
SAMBA, I. et BOUSSO, Y. D., A. (2014). Les langues nationales en contexte scolaire au Sénégal: pour une acceptabilité sociale. African Educational Development Issues, 5, 71-96.

SANGARE, S. et DIARRA, I. (1997). Effets de la participation communautaire sur l'accès et la qualité de l'éducation de base: le cas du Mali (rapport de recherche). Bamako, Mali: ROCARE. Repéré à http://www.ROCARÉ.org/part_qualite_acces.PDF

SENE, P. A. (2008). Évaluation de la pratique des enseignants en matière de TIC dans les écoles au Sénégal. Dans K. Touré, T. M. S. Tchombe et T. Karsenti (dir.), ICT and Changing Mindsets in Education (p. 125-136). Bamenda, Cameroun: Langaa.

SÉNOU, B. M., SOGLO, M. A., QUENUM, B. M et OKAN, M. (2010). Qualité de l'enseignement supérieur et marché du travail au Bénin (rapport de recherche). Bamako, Mali: ROCARE.

SILUÉ, N. O. et N’DJORÉ, B. A. A. Y. (2016). Intégration des écoles confessionnelles islamiques dans le système éducatif. Dans F.-J. Azoh et T. Goin-Bi (dir.), Nouveaux défis de l'éducation en Côte d'Ivoire (p. 35-90). Abidjan, Côte d'Ivoire: Éburnie.

SY, A. et TRAORE, M. (1997). Alphabétisation accélérée pour jeunes non-scolarisés (rapport de recherche). Bamako, Mali: ROCARE. Repéré à http://www.ernwaca. org/biblio/opac_css/index.php?lvl=index

TCHAGNAOU, A. et BAOUTOU, A. (2016). La formation initiale des enseignants au service d'une éducation de qualité au secondaire 1 au Togo. Revue de vulgarisation du ROCARE, 1, 19-24.

TOUNKARA, B., FOMBA, C.-O. et FANÉ, D. (2001). Évaluation de la qualité de l'éducation dans des écoles communautaires gérées par Africare et ONG partenaires (rapport de recherche). Bamako, Mali: ROCARE. Repéré à http:// www.ernwaca.org/biblio/opac_css/index.php?lvl=index

TSIGBE, K. N. (2010). Contribution des TIC au développement et à la qualité de l'enseignement supérieur à l'université de Lomé (Togo). African Education Development Issues, 1, 132-154.

ORGANISATION DES NATIONS UNIES POUR L'ÉDUCATION, LA SCIENCE ET LA CULTURE (UNESCO). (2004). Éducation pour tous: l'exigence de qualité. Rapport mondial de suivi sur l'EPT 2005. Paris, France: Auteur. Repéré à http:// unesdoc.unesco.org/images/0013/001374/137403f.pdf 
ORGANISATION DES NATIONS UNIES POUR L'ÉDUCATION, LA SCIENCE ET LA CULTURE (UNESCO). (2012). L'éducation et la culture de la paix au cour de la participation de la Directrice générale à l'Assemblée générale des Nations Unies. Repéré à http://www.unesco.org/new/fr/media-services/single-view/news/leducation_ et_la_culture_de_la_paix_au_coeur_de_la_parti/

VERSPOOR, A. (2005). Le défi de l'apprentissage: améliorer la qualité de l'éducation de base en Afrique subsaharienne. Paris, France: L'Harmattan.

WALET, A., BOCOUM, I., BAMBA, Y. et FAYE, H. (2010). Les déterminants pédagogiques de la réussite scolaire: le cas des écoles du Centre d'Animation Pédagogique de Torokorobougou dans le District de Bamako (rapport de recherche). Bamako, Mali: ROCARE. Repéré à http://www.ernwaca.org/biblio/ opac_css/index.php?lvl=index 